\begin{tabular}{|c|l|}
\hline Title & First-order photon interference of a single photon from a single quantum dot \\
\hline Author(s) & Ekuni, S.; Nakajima, H.; Sasakura, H.; Suemune, I.; Kumano, H. \\
\hline Citation & $\begin{array}{l}\text { Physica E: Low-dimensional Systems and Nanostructures, 42(10), 2536-2539 } \\
\text { https://doi.org/10.1016/.physe.2009.11.029 }\end{array}$ \\
\hline Issue Date & 2010-09 \\
\hline Doc URL & http://hdl.handle.net/2115/45365 \\
\hline Type & article (author version) \\
\hline File Information & 294_suemune.pdf \\
\hline
\end{tabular}

Instructions for use 


\title{
First-order photon interference of a single photon from a single quantum dot
}

\author{
S. Ekuni ${ }^{1}$, H. Nakajima ${ }^{1}$, H. Sasakura ${ }^{1}$, I. Suemune ${ }^{1,2}$, and H. Kumano ${ }^{1,2, *}$ \\ ${ }^{1}$ RIES, Hokkaido University, Sapporo 001-0021, Japan \\ ${ }^{2}$ Japan Science and Technology Corporation (CREST), Saitama 332-0012, Japan 332-0012, Japan
}

\begin{abstract}
Photon interference indicating wave-like nature of a single photon emitted from a single quantum dot is demonstrated. Photon state as a superposition of two orthogonal linear polarization modes is prepared inside a solid-state single photon source, which causes the first-order interference analogous to the Young's double slit experiment. The lack of which-mode information is essential for observing the single photon interference.
\end{abstract}

PACS: 42.25.Hz; 42.50.Ct; 42.50.Dv; 71.35.-y

Keywords: Quantum dots; Single photon interference; Double slit experiment; Which-path information

\section{Introduction}

As stated by Feynman, double slit interference is a phenomenon "which has in it the heart of quantum mechanics; in reality it contains the only mystery" of the quantum theory[1]. So far, a wide variety of the Young's double slit experiments have been demonstrated for massive particles such as neutrons [2], atoms[3], single electrons[4] and single molecules[5], and also for the massless photon with attenuated coherent light[6] and with single photons from a color center in a diamond nanocrystal [7]. These pioneering attempts have successfully revealed the mystery based on wave-particle duality as a manifestation of the quantum complementarity [8,9] belonging to the quantum mechanical objects. In all these experiments, two modes to generate interference are prepared outside each source of the objects, and only a few experiments have clarified which-path interference in solid-state systems with two electron modes [10,11]. Furthermore, single-photon Young's double slit type interference has not so far been demonstrated in solid-state systems.

In this paper, first-order single photon interference between two polarization modes is demonstrated with a solid-state single photon emitter. The orthogonal linear polarization modes are prepared in a non-degenerate neutral exciton states in the single quantum dot (QD). Interference between the two polarization modes appears only when the two modes are superposed without "which-mode" information by its transition energies.

\footnotetext{
${ }^{*}$ Corresponding author. Tel. \& Fax: +81-11-706-9334

E-mail address: kumano@es.hokudai.ac.jp (H. Kumano)
} 


\section{Experimental}

An $\mathrm{In}_{0.75} \mathrm{Al}_{0.25} \mathrm{As}$ QDs sample was grown on a semi-insulating (001)-GaAs substrate by molecular-beam epitaxy. The QDs were prepared in Stranski-Krastanow (S-K) growth mode on $\mathrm{Al}_{0.3} \mathrm{Ga}_{0.7} \mathrm{As}$ layers and were sandwiched with the $\mathrm{Al}_{0.3} \mathrm{Ga}_{0.7}$ As layers. The topmost surface was terminated with a GaAs cap layer. After the growth, the sample was etched into mesa structures with diameters of $\sim 150 \mathrm{~nm}$ for isolating single QD from the dot ensemble with the density of around $5 \times 10^{10} \mathrm{dots} / \mathrm{cm}^{2}$. Further details of this sample preparation are described in Ref. 12. For a single dot spectroscopy, a continuous-wave Ti: sapphire laser was used as a linearly polarized excitation source. An objective lens with the numerical aperture of 0.42 focused the laser beam on one of the mesa structures and collected photoluminescence (PL) emitted from the mesa.

In order to discuss single photon interference between two polarization modes, luminescence collected under non-resonant excitation $(1.687 \mathrm{eV})$ was analyzed by a half wave plate (HWP) and a following linear polarizer (LP1) placed in front of a $0.64-\mathrm{m}$ triple-grating spectrometer equipped with a liquid-nitrogen cooled Si charge-coupled-device (CCD) detector (energy resolution of this detection system $<5 \mu \mathrm{eV}$ ) as depicted in Fig. 1 (c). Transmission axis of the LP1 is set vertical in the laboratory frame and aligned with the crystalline axis of the sample. Two quantum dots (QD A and QD B) with different exciton fine structure splitting (FSS) and spectral linewidth were studied at $20 \mathrm{~K}$. Prior to the present study, generation of single photons from both QDs has been confirmed independently by the photon correlation measurements [13-15]. Throughout this work, measurements were carried out under weak excitation condition in below $50 \mu \mathrm{W}$, above which a second order photon correlation function at zero delay $\mathrm{g}^{(2)}(0)$ showed noticeable degradation indicating multi-photon emission [16]. Therefore only single photon regime is responsible for the results discussed hereafter.

\section{Results and discussion}

In QD A, four emission lines located at $1.5976 \mathrm{eV}$ (neutral exciton: $\mathrm{X}^{0}$ ), $1.5964 \mathrm{eV}$ (neutral bi-exciton: $\mathrm{XX}^{0}$ ), $1.5966 \mathrm{eV}$ (negatively charged exciton: $\mathrm{X}^{-}$), and $1.6009 \mathrm{eV}$ (positively charged exciton: $\mathrm{X}^{+}$) were dominant and the origins of each line were closely investigated by diversified experiments [16]. In this paper, in order to discuss an effect of which-mode information on a single photon interference in a rectilinear polarization basis, the $\mathrm{X}^{0}$ emission is examined. PL spectra of the $\mathrm{X}^{0}$ measured with vertical $(\mathrm{V})$ and horizontal $(\mathrm{H})$ polarizations are shown in Fig. 1(a) and the solid lines are fitted results with the Lorentzian function. Clear energy splitting between these two spectra of $110 \mu \mathrm{eV}$ is found, which corresponds to the FSS due to the anisotropy of confinement potential [17]. The FSS is larger than the spectral linewidth (full width at half maximum) of 78 $\mu \mathrm{eV}$, and therefore the two spectra are well separated in energy.

Linear-polarization dependence of emitted photons is displayed as a contour plot in Fig. 2(a). In this figure the vertical axis indicates polarization rotation angle $2 \theta,(\theta$ is defined as a fast axis 
angle of the HWP measured from the vertical), while the horizontal axis shows photon energy. Hereafter, the angle dependences will be discussed in terms of $2 \theta$. It is found that emitted photons are linearly polarized and the transition energy of the $\mathrm{X}^{0}$ line is clearly two-valued, therefore the two linear polarization modes $\mathrm{V}$ and $\mathrm{H}$ can be distinguishable by their transition energies (which-mode information). On the other hand, QD B has less and less which-mode information. Figure 1(b) shows PL spectra of the QD B measured with V and H polarizations and their Lorentzian fit (solid curves). In the QD B, FSS and the linewidths are $30 \mu \mathrm{eV}$ and $100 \mu \mathrm{eV}$, respectively, therefore, the large spectral overlap make the transition energy labeling to the two polarization modes indefinite.

Between these two QDs under investigation, integrated PL intensity over the whole spectral region of the $\mathrm{X}^{0}$ emission is quite different. In the QD A, the integrated intensity remains constant against the detection polarization angle as shown in Fig. 2(d), while the QD B exhibits periodic change depending on the detection polarizations as shown in Fig. 3(a). In addition, in the QD B, $\pi / 4$ phase shift from the vertical is obvious, where the intensity is maximized at $\pi / 4+n \pi$ ( $n$ : integer) denoted as $\mathrm{D}$ and minimized at $3 \pi / 4+n \pi$ denoted as $\mathrm{D}^{*}$. The intensity difference between $\mathrm{D}$ and $\mathrm{D}^{*}$ readily indicates that the photon state can not be attributed to a single mode ( $\mathrm{V}$ or $\left.\mathrm{H}\right)$ because, if it is the case, the projection of the state vector to the orthogonal diagonal directions $\mathrm{D}$ and $\mathrm{D}^{*}$ always yields to the equal intensity. Thus the contribution of a superposition state of the two polarization mode is inferred. Further analyses of the observed intensity for both QDs are carried out below:

Assuming the same amplitude for both polarization modes ensured by Figs. 1(a) and (b), the single photon state generated by the radiative recombination of a neutral exciton in a single QD can be described, for two extreme cases, as (i) $\rho=1 / 2(|V\rangle\langle V|+| H\rangle\langle H|)$ for mixed states composed of the two exclusive polarization modes $|\mathrm{V}\rangle$ and $\mid \mathrm{H}>$, and (ii) $\rho=|\psi\rangle\langle\psi|$ for pure state $|\psi\rangle=\frac{1}{\sqrt{2}}(|V\rangle+|H\rangle)$, which is a superposition of two polarization modes. Jones matrix $\mathrm{P}(\pi)$ for the HWP with its fast axis in the vertical direction is given by $\left(\begin{array}{cc}1 & 0 \\ 0 & e^{i \pi}\end{array}\right)$ under $|V\rangle=(1,0)^{T}$ and $|H\rangle=(0,1)^{T}$ basis [18]. Since the function of the LP1 is described by a projection operator $\Lambda_{\mathrm{V}}=|\mathrm{V}\rangle\langle\mathrm{V}|$, photon field operator $\mathrm{E}(\theta)$ via these HWP and LP1 is given by $\Lambda_{\mathrm{V}} \cdot \mathrm{R}(\theta) \mathrm{P}(\pi) \mathrm{R}(\theta)^{-1}$, where $\mathrm{R}(\theta)$ is a rotation matrix expressed by $\left(\begin{array}{cc}\cos \theta & -\sin \theta \\ \sin \theta & \cos \theta\end{array}\right)$. In the mixed photon state, integrated PL intensity $I(\theta)$ given by $\operatorname{Tr}\left\{\rho \mathrm{E}(\theta)^{\dagger} \mathrm{E}(\theta)\right\}$ leads to the constant value of $1 / 2$ independent of $\theta$, which agrees well with the experimental result for the QD A (Fig. 2(d)). On the other hand, for the 1 photon 2 mode state (pure state) $I(\theta)$ becomes $\frac{1}{2}[1+\cos 2(2 \theta-\pi / 4)]$, which reproduces the experimental polarization angle dependence of the QD B including modulation period of $\pi$ and 
phase shift of $\pi / 4$ as shown in Fig. 3(a).

With respect to the above argument, information on the polarization mode of a detected photon is present if linewidth $<$ FSS. In this case, the photon state is reduced to the mixed state and the interference disappears. On the other hand, if linewidth > FSS, spectral overlap measured under the two polarizations are large enough not to label the two polarization modes by their transition energies. Then a superposition state is formed, which leads to the first-order two-mode interference indicating the wave-like nature of a photon.

In order to elucidate the contribution of the photon interference, additional linear polarizer $\mathrm{LP} 2(\mathrm{~V} / \mathrm{H})$ is inserted prior to the HWP with its transmission axis being set to $\mathrm{V}$ or $\mathrm{H}$. Contour plots with each PL2(V) and PL2(H) are given in Figs. 2(b) and (c), respectively, and their sum is displayed in Fig. 2(e). In the QD A, the summed one-mode intensity well reproduces the integrated intensity obtained without the PL2. Then the contribution of the interference is, if any, very small. On the other hand, in the QD B, the summed intensity fails to explain the sinusoidal intensity change. This is because the summed one-mode intensity does not include the interference contribution that is essential for the two-mode state prepared when linewidth > FSS.

\section{Summary}

In summary, wave-like nature of a photon, i.e., two-mode interference of a single photon is observed with non-degenerate neutral exciton states in a single quantum dot. In this study, the neutral exciton states define the single photon polarization modes, and the energy relaxation process in solid state system serves as a mode distributor. As expected, parallel relations with the Young's double slit experiment do exist, i.e, two polarization modes correspond to two spatial modes specified by wavevectors from each slit to one position on a screen, and the detection intensity depends on $2 \theta$ instead of screen position. The one-photon two-mode interference takes place only under the condition that the polarization mode of emitted photon is indistinguishable in energy, in other words, the which-mode information is absent.

\section{Acknowledgement}

The authors would like to acknowledge Dr. H. Z. Song, S. Hirose and M. Takatsu for the sample preparation, and S. Muto, S. Adachi, M. Jo and R. Kaji for fruitful discussions. This work was supported in part by the Grant-in-Aid for Scientific Research (A)(2), No. 21246048, Young Scientists (A), No. 2168102009, and Hokkaido Innovation Through Nanotechnology Supports (HINTs) from the Ministry of Education, Culture, Sports, Science and Technology. 


\section{References}

[1] R. P. Feynman, R. B. Leighton, and M. Sands, The Feynman Lectures on Physics (Wesley, 1965), Vol. 3, Ch. 1.

[2] A. Zeilinger, R. Gähler, C. G. Shull, W. Treimer and W. Mampe, Rev. Mod. Phys. 60, 1067 (1988).

[3] O. Carnal and J. Mlynek, Phys. Rev. Lett. 66, 2689 (1991).

[4] A. Tonomura, J. Endo, T. Matsuda, T. Kawasaki, and H. Ezawa, Am. J. Phys. 57, 117 (1989).

[5] O. Nairz, M. Arndt, and A. Zeilinger, Am. J. Phys. 71, 319 (2002).

[6] R. L. Pfleegor and L. Mandel, Phys. Rev. 159, 1084 (1967).

[7] V. Jacques, E. Wu, T. Toury, F. Treussart, A. Aspect, P. Grangier, and J.-F. Roch. Eur. Phys. J. D 35, 561 (2005).

[8] M. O. Scully, B. Englert, and H. Walther, Nature 351, 111 (1991).

[9] V. Jacques, E Wu, F. Grosshans, F. Treussart, P. Grangier, A. Aspect, and J. F. Roch, Science 315, 966 (2007).

[10] R. Schuster, E. Buks, M. Heiblum, D. Mahalu, V. Umansky, and H. Shtrikman, Nature 385, 417 (1997).

[11] K. Furuya, Y. Ninomiya, N. Machida, and Y. Miyamoto, Phys. Rev. Lett. 91, 216803 (2003).

[12] H. Sasakura, S. Adachi, S. Muto, H. Z. Song, T. Miyazawa, and T. Usuki, Jpn. J. Appl. Phys., 43, 2110 (2004).

[13] S. Kimura, H. Kumano, M. Endo, I, Suemune, T. Yokoi, H. Sasakura, S. Adachi, S. Muto, H. Z. Song, S. Hirose, and T. Usuki, Jpn. J. Appl. Phys. 44, L793-L796 (2005).

[14] S. Kimura, H. Kumano, M. Endo, I, Suemune, T. Yokoi, H. Sasakura, S. Adachi, S. Muto, H. Z. Song, S. Hirose, and T. Usuki, phys. stat. sol. (c) 2, 3833-3837 (2005).

[15] H. Kumano, S. Kimura, M. Endo, I. Suemune, H. Sasakura, S. Adachi, S. Muto, H. Z. Song, S. Hirose, and T. Usuki, Physica E 32, 144-147 (2006).

[16] H. Kumano et al., Journal of Nanoelectron. and Optoelectron. 1, 39-51 (2006).

[17] V. D. Kulakovskii, G. Bacher, R. Weigand, T. Kümmell, A. Forchel, E. Borovitskaya, K. Leonardi, and D. Hommel, Phys. Rev. Lett. 82, 1780 (1999).

[18] E. Hecht, Optics, 4th Ed, Chapter 8, Addison Wesley (2002). 


\section{Figure captions}

Fig. 1

(color on line) (a), (b) Emission lineshape of the $\mathrm{X}^{0}$ in (a) QD A and (b) QD B for both polarization modes $\mid V>$ and $\mid H>$. (c) Schematic of the experimental setup. A rotatable HWP and linear polarizer (LP1), followed by a triple-grating and multi-channel detector are used for photon detection. In some measurements, additional linear polarizer (LP2) is also introduced.

Fig. 2

(color on line) (a) Contour plot of the detection intensity as a function of photon energy and polarization rotation angle $2 \theta$ for the $\mathrm{X}^{0}$ in the QD A. (b), (c) Contour plots for the one-mode photon states (b) V and (c) H measured with the LP2. (d) Integrated PL intensity over the whole spectral region of the $X^{0}$ emission without the LP2. (e) Sum of two one-mode intensities LP2(V) and LP2(H) obtained from (b) and (c), respectively.

Fig. 3

(color on line) Results of the corresponding measurements to Figs. 2(d) and (e) for QB B. (a) Normalized intensity as a function of $2 \theta$ for the $\mathrm{X}^{0}$ in the QD B. Detection polarization directions are illustrated. Solid curve is a fitted result with $\frac{1}{2}\left[1+\nu \cos 2\left(2 \theta+\theta_{0}\right)\right]$, where the resultant visibility $\checkmark$ is 0.14 . (b) Sum of two one-mode intensities LP2(V) and LP2(H). 


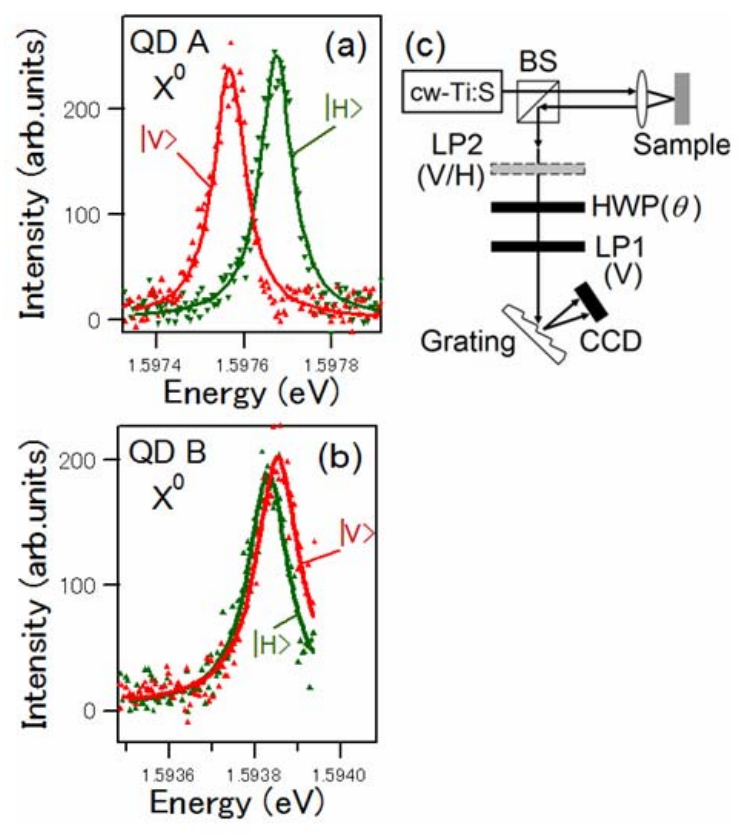

S. Ekuni et al., Figure 1
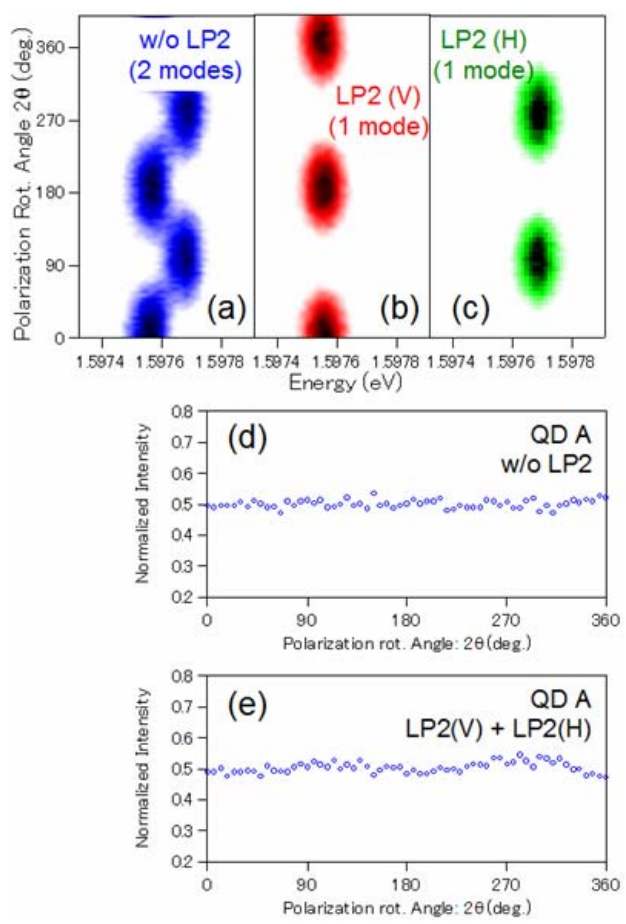

S. Ekuni et al., Figure 2 

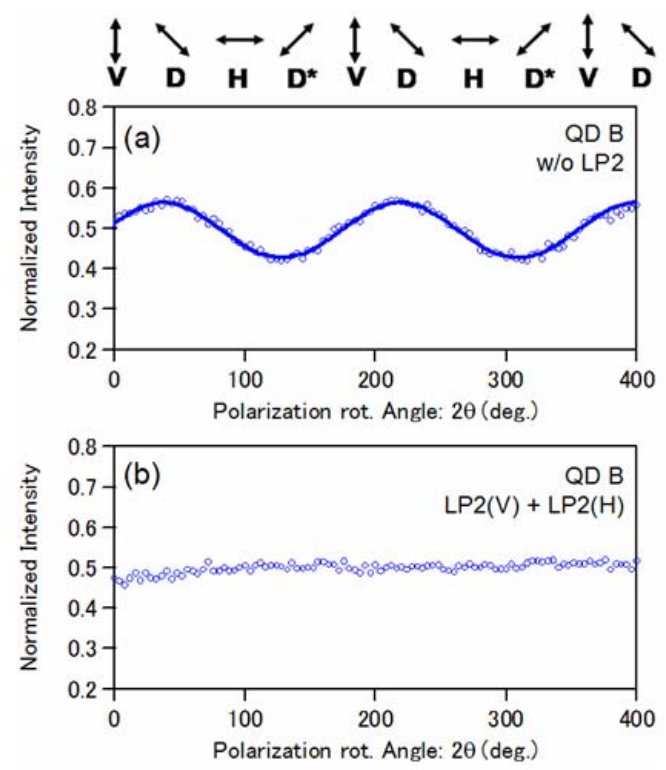

S. Ekuni et al., Figure 3 